\title{
Fuel Provider Evaluation Using a Combined Decision Aid Model
}

\author{
Mehtap Dursun ${ }^{1, *}$, Nazli Goker ${ }^{1}$ \\ ${ }^{1}$ Galatasaray University, Industrial Engineering Department, 34349 Ortakoy, Istanbul, Turkey
}

\begin{abstract}
Energy is a basic need for achieving quality of life and the level of welfare. Chemical or nuclear energy are released as heat in the wake of a reaction between fuel and other substances. Heat is used for warming, cooking, or industrial processes. This paper presents an integrated multiple criteria decision making framework for fuel provider selection in food industry. Evaluation factors are named as lead time, reliability, sustainability, cost, service quality, location and warranties. To demonstrate the robustness of the application, a numerical illustration is provided by conducting a case study in food industry of Turkey.
\end{abstract}

\section{Introduction}

Fuel is a material that can react with other substances in order to release chemical or nuclear energy as heat or to be used for work. The heat energy released by reactions of fuels is converted into mechanical energy via a heat engine. Heat is valued for warmth, cooking, or industrial processes, as well as the illumination that comes with combustion. Over the years, energy has been one of the most important parts of the human life. One shall note that wood is the first fuel which was used with the aim of lighting and cooking. Wood and charcoal have been used intentionally till coal replaced wood. Then, oil has been discovered as a competitor with coal in the fuel industry. With the discovery of natural gas, fossil fuels and fuel industry developed a new identity. By means of these biomass energies such as firewood, charcoal, dung, agricultural residues; new renewable energy technologies begin to evolve in the 21 th century [1].

This study aims to identify the most suitable fuel provider alternative by employing linguistic hierarchies and TOPSIS method. Over the last decade, researchers have contributed to the provider selection by proposing multi-criteria decision making approaches. $\mathrm{Wu}$ and Chien [2] proposed a decision framework to assess outsourcing providers and to solve order allocation decision problem. Büyüközkan et al. [3] introduced a 2additive choquet integral method to fourth party logistics service provider selection problem. They carried out a case study in a logistic firm that performs in Turkey. Kahraman et al. [4] ranked IT service providers for a furniture company in Konya, Turkey. Wan et al. [5] identified the most appropriate logistics outsourcing provider by employing an intuitionistic fuzzy linear programming method. Govindan et al. [6] determined 3PL provider selection criteria to enable managers in automotive industry to obtain competitive advantage. Wang et al. [7] assessed the contractors in logistics outsourcing. Rajaeian et al. [8] provided a literature survey on information technology (IT) outsourcing by applying MCDM, optimization and simulation methods that support IT outsourcing decision process.

The rest of the study is organized as follows. Section 2 and 3 outline the 2-tuple linguistic representation model and linguistic hierarchies, respectively. Section 3 presents TOPSIS method. The case study, which is conducted in food industry of Turkey, is presented in Section 5. Finally, concluding remarks and future research directions are delineated in the last section.

\section{2-tuple linguistic representation model}

The 2-tuple linguistic model that was introduced by Herrera and Martínez [9] is based on the concept of symbolic translation. It is utilized to represent the linguistic assessment information by means of a 2-tuple that is composed of a linguistic term and a number. It can be denoted as $\left(s_{i}, \alpha\right)$ where $s_{i}$ denotes the linguistic label of the described linguistic term set $S_{T}$, and $\alpha$ is a numerical value indicating the symbolic translation.

Important definitions are given in the following to operate with the 2-tuples without loss of information.

Definition 1 [10]: Let $L=\left(\gamma_{0}, \gamma_{1}, \ldots, \gamma_{g}\right)$ be a fuzzy set described in $S_{T}$. A transformation function $\boldsymbol{\chi}$ that transforms $L$ into a numerical value in the interval of granularity of $S_{T},[0, g]$ is given as

$$
\begin{aligned}
& \chi: F\left(\mathrm{~S}_{T}\right) \rightarrow[0, g], \\
& \chi\left(F\left(\mathrm{~S}_{T}\right)\right)=\chi\left(\left\{\left(s_{j}, \gamma_{j}\right), j=0,1, \ldots, g\right\}\right)=\frac{\sum_{j=0}^{g} j \gamma_{j}}{\sum_{j=0}^{g} \gamma_{j}}=\beta .
\end{aligned}
$$

where $F\left(S_{T}\right)$ is the set of fuzzy sets defined in $S_{T}$.

\footnotetext{
Corresponding author: $\underline{\text { mdursun } @ \text { gsu.edu.tr }}$
} 
Definition 2 [9]: Let $S=\left\{s_{0}, s_{1}, \ldots, s_{g}\right\}$ be a linguistic term set and $\beta \in[0, g]$ a value supporting the result of a symbolic aggregation operation, then the 2-tuple that expresses the equivalent information to $\beta$ is obtained with the following function:

$\Delta:[0, g] \rightarrow S \times[-0.5,0.5)$,

$\Delta(\beta)=\left\{\begin{array}{lc}s_{i}, & i=\operatorname{round}(\beta) \\ \alpha=\beta-i, & \alpha \in[-0.5,0.5),\end{array}\right.$

where 'round' is the usual round operation, $s_{i}$ has the closest index label to ' $\beta$ ' and ' $\alpha$ ' is the value of the symbolic translation.

Proposition 1 [9]: Let $S=\left\{s_{0}, s_{1}, \ldots, s_{g}\right\}$ be a linguistic term set and $\left(s_{i}, \alpha\right)$ be a 2 -tuple. There is a $\Delta^{-1}$ function, such that, from a 2-tuple it returns its equivalent numerical value $\beta \in[0, g] \subset \mathfrak{R}$. This function is defined as

$$
\begin{aligned}
& \Delta^{-1}: S \times[-0.5,0.5) \rightarrow[0, g] \\
& \Delta^{-1}\left(s_{i}, \alpha\right)=i+\alpha=\beta .
\end{aligned}
$$

\section{Linguistic hierarchies}

The concept of linguistic hierarchies was proposed by Cordon et al. [11] to design hierarchical systems of linguistic rules, then it was utilized to enhance precision of computing with words in the multi-granular linguistic information contexts [10]. A linguistic hierarchy is a set of levels, where each level is a linguistic term set with different granularity to the rest of levels of the hierarchy. Each level belonging to a linguistic hierarchy is denoted as $l(t, n(t))$, where $t$ indicates the level of the hierarchy, and $n(t)$ is the granularity of the linguistic term set of the level $t$ [10]. A linguistic hierarchy, $L H$, can be defined as the union of all levels $t$ as $L H=\bigcup_{t} l(t, n(t))$.

The linguistic term set of level $t+1$ is obtained from its predecessor as [10]

$$
L(t, n(t)) \rightarrow L(t+1,2 . n(t)-1)
$$

Linguistic hierarchies are used to avoid the problem of loss of information that occurs in the unification phase of multigranular linguistic information. The transformation function between linguistic terms in any level of the hierarchy is defined as

$$
\begin{aligned}
& T F_{t^{\prime}}^{t}: l(t, n(t)) \rightarrow l\left(t^{\prime}, n\left(t^{\prime}\right)\right) \\
& T F_{t^{\prime}}^{t}\left(s_{i}^{n(t)}, \alpha^{n(t)}\right)=\Delta\left(\frac{\Delta^{-1\left(s_{i}^{n(t)}, \alpha^{n(t)}\right)\left(n\left(t^{\prime}\right)-1\right)}}{n(t)-1}\right)
\end{aligned}
$$

The transformation function is bijective, which guarantees the transformations are performed without loss of information [10].

\section{TOPSIS method}

The technique for order preference by similarity to ideal solution (TOPSIS) proposed Hwang and Yoon [12] is one of the well-known methods for classical multiattribute decision making. TOPSIS is based upon the assumption that the chosen alternative should have the shortest distance from the ideal solution and the farthest from the anti-ideal solution.

The stepwise representation of the TOPSIS is given below.

Step 1. Identify the alternatives $A_{i},(i=1,2, \ldots, m)$, and required selection criteria $C_{j},(j=1,2, \ldots, n)$.

Step 2. Construct the decision matrices that denote the importance weight of criteria, and the ratings of alternatives with respect to criteria.

Step 3. Normalize the decision matrix to obtain unit-free and comparable criteria values as

$r_{i j}=\frac{x_{i j}}{\sqrt{\sum_{i=1}^{m} x_{i j}^{2}}} i=1,2, \ldots, m ; j=1,2, \ldots, n$

where $r_{i j}$ denotes the normalized value of $x_{i j}, m$ is the number of alternatives, $n$ is the number of criteria.

Step 4. Calculate the weighted normalized decision matrix. The weighted normalized value $v_{i j}$ is calculated as

$v_{i j}=w_{j} r_{i j}, i=1,2, \ldots, m ; j=1,2, \ldots, n$

where $w_{j}$ is the weight of the $j$ th criterion, and $\sum_{j=1}^{n} w_{j}=1$

Step 5. Define the ideal solution, $A^{*}$, and the anti-ideal solution, $A^{-}$. The $A^{*}$ and $A^{-}$are defined in terms of the weighted normalized values as shown in Eqs. (8) and (9), respectively.

$$
\begin{aligned}
A^{*} & =\left\{v_{1}^{*}, v_{2}^{*}, \ldots, v_{n}^{*}\right\} \\
& =\left\{\left(\max _{i} v_{i j} \mid j \in J_{1}\right),\left(\min _{i} v_{i j} \mid j \in J_{2}\right)\right\}, \\
A^{-} & =\left\{v_{1}^{-}, v_{2}^{-}, \ldots, v_{n}^{-}\right\} \\
& =\left\{\left(\min _{i} v_{i j} \mid j \in J_{1}\right),\left(\max _{i} v_{i j} \mid j \in J_{2}\right)\right\}
\end{aligned}
$$

where $J_{1}$ is the set of benefit-related criteria for which the greater the performance value the more its preference, $J_{2}$ is the set of cost-related criteria for 
which the greater the performance value the less its preference.

Step 6. Calculate the separation measures using the $n$ dimensional Euclidean distance. The separation of each alternative from the ideal solution is given as

$$
D_{i}^{*}=\sqrt{\sum_{j=1}^{n}\left(v_{i j}-v_{j}^{*}\right)^{2}}, \quad i=1,2, \ldots, m
$$

Similarly, the separation from the anti-ideal solution is given as

$$
D_{i}^{-}=\sqrt{\sum_{j=1}^{n}\left(v_{i j}-v_{j}^{-}\right)^{2}}, \quad i=1,2, \ldots, m
$$

Step 7. Calculate the relative closeness to the ideal solution as in Eq. (12).

$$
C_{i}^{*}=\frac{D_{i}^{-}}{D_{i}^{*}+D_{i}^{-}}, \quad i=1,2, \ldots, m
$$

Step 8. Rank the alternatives according to $C_{i}^{*}$ values in descending order. Identify the alternative with the highest $C_{i}^{*}$ as the best alternative.

\section{Case study}

To illustrate the application of the proposed decision making approach for fuel provider selection, a case study conducted in food industry of Turkey, is introduced. The case company performs in Turkish food sector, and wants to provide cooking fuel from a third party provider. Evaluation criteria are determined and assessed by literature survey and opinions of three employees who works in the case company for minimum four years. Seven criteria for fuel provider problem are defined as
$C_{1}$ : Lead time
$C_{2}$ : Reliability
$C_{3}$ : Sustainability
$C_{4}$ : Cost
$C_{5}$ : Service quality
$C_{6}$ : Location
$C_{7}$ : Warranties

The evaluation is conducted by a committee of three decision-makers $\left(D M_{1}, D M_{2}, D M_{3}\right)$. The linguistic hierarchy $L H=\bigcup_{t} l(1,3)$, shown in Table 1, is considered as multi-granular linguistic context, since the granularity of its linguistic term sets are very common in decisionmaking problems.
Table 1. Linguistic hierarchy $L H=\bigcup_{t} l(1,3)$

\begin{tabular}{|c|c|}
\hline$l(1,3)$ & $\left(s_{0}^{3}, s_{1}^{3}, s_{2}^{3}\right)$ \\
\hline$l(2,5)$ & $\left(s_{0}^{5}, s_{1}^{5}, s_{2}^{5}, s_{3}^{5}, s_{4}^{5}\right)$ \\
\hline$l(3,9)$ & $\left(s_{0}^{9}, s_{1}^{9}, s_{2}^{9}, s_{3}^{9}, s_{4}^{9}, s_{5}^{9}, s_{6}^{9}, s_{7}^{9}, s_{8}^{9}\right)$ \\
\hline
\end{tabular}

The linguistic term set $l(2,5)$ is indicated as linguistic terms set to unify the multi-granular linguistic information given by the experts. In the decision process, equal weights are appointed to decision-makers. Hence, the unified evaluations of decision-makers are aggregated by incorporating 2-tuple mean operator, and the aggregated data related to agile supplier selection problem are given in Table 2.

Table 2. Aggregated data related to agile supplier selection problem

\begin{tabular}{|l|c|c|c|c|c|}
\hline & $\boldsymbol{A}_{\mathbf{1}}$ & $\boldsymbol{A}_{\mathbf{2}}$ & $\boldsymbol{A}_{\mathbf{3}}$ & $\boldsymbol{A}_{\mathbf{4}}$ & Weight \\
\hline $\boldsymbol{C}_{\mathbf{1}}$ & $\left(s_{4}^{5},-0.33\right)$ & $\left(s_{1}^{5}, 0.33\right)$ & $\left(s_{2}^{5},-0.17\right)$ & $\left(s_{4}^{5},-0.17\right)$ & $\left(s_{1}^{5},-0.17\right)$ \\
\hline $\boldsymbol{C}_{\mathbf{2}}$ & $\left(s_{3}^{5},-0.5\right)$ & $\left(s_{4}^{5},-0.33\right)$ & $\left(s_{4}^{5},-0.33\right)$ & $\left(s_{1}^{5},-0.17\right)$ & $\left(s_{4}^{5},-0.33\right)$ \\
\hline $\boldsymbol{C}_{\mathbf{3}}$ & $\left(s_{3}^{5},-0.5\right)$ & $\left(s_{4}^{5},-0.5\right)$ & $\left(s_{4}^{5},-0.33\right)$ & $\left(s_{4}^{5},-0.33\right)$ & $\left(s_{3}^{5}, 0.17\right)$ \\
\hline $\boldsymbol{C}_{\mathbf{4}}$ & $\left(s_{4}^{5},-0.5\right)$ & $\left(s_{1}^{5}, 0.33\right)$ & $\left(s_{3}^{5},-0.33\right)$ & $\left(s_{3}^{5}, 0.17\right)$ & $\left(s_{4}^{5},-0.5\right)$ \\
\hline $\boldsymbol{C}_{\mathbf{5}}$ & $\left(s_{4}^{5},-0.33\right)$ & $\left(s_{4}^{5},-0.17\right)$ & $\left(s_{2}^{5}, 0.33\right)$ & $\left(s_{1}^{5},-0.17\right)$ & $\left(s_{1}^{5},-0.17\right)$ \\
\hline $\boldsymbol{C}_{\mathbf{6}}$ & $\left(s_{4}^{5},-0.33\right)$ & $\left(s_{1}^{5},-0.17\right)$ & $\left(s_{2}^{5}, 0.33\right)$ & $\left(s_{4}^{5},-0.17\right)$ & $\left(s_{3}^{5}, 0.17\right)$ \\
\hline $\boldsymbol{C}_{7}$ & $\left(s_{2}^{5}, 0.33\right)$ & $\left(s_{2}^{5},-0.17\right)$ & $\left(s_{4}^{5},-0.17\right)$ & $\left(s_{4}^{5},-0.17\right)$ & $\left(s_{4}^{5},-0.33\right)$ \\
\hline
\end{tabular}

By employing TOPSIS method, the final ranking of alternatives is obtained as in Table 3 .

Table 3. Ranking of alternatives

\begin{tabular}{|l|c|c|c|}
\hline & $D^{*}$ & $D-$ & $C^{*}$ \\
\hline$A_{1}$ & 5.317 & 0.786 & 0.128 \\
\hline$A_{2}$ & 5.368 & 0.980 & 0.154 \\
\hline$A_{3}$ & 5.218 & 0.857 & 0.141 \\
\hline$A_{4}$ & 5.272 & 0.841 & 0.137 \\
\hline
\end{tabular}

\section{Conclusions}

Fuel provider selection problem, which contains several individual factors including vagueness and imprecision, may be thought as a highly important group decisionmaking problem. In this study, a fuzzy multi-criteria decision making approach which combines 2-tuple fuzzy linguistic modeling, linguistic hierarchies, and TOPSIS is introduced. The developed approach aims to manage multi-granular linguistic information, allows decision makers to use different semantic types, and copes with 
loss of information which may be occur due to the classical MCDM methods.

Lead time, reliability, sustainability, cost, service quality, location and warranties are considered as evaluation criteria. A numerical example, which illustrates the application, is provided by conducting a case study in food sector of Turkey. Future research may focus on multi-criteria decision problems with the presence of interdependence/interactions among criteria which influence the ranking process.

\section{Acknowledgement}

This work has been financially supported by Galatasaray University Research Fund 19.402.004.

\section{References}

1. A. Isler, F. Karaosmanoglu, Istanbul PreConference Workshop on Energy and Poverty: Clean Cooking Fuels, 16-17 June (2008).

2. J.Z. Wu, C.F. Chien, Modeling strategic semiconductor assembly outsourcing decisions based on empirical settings. OR Spectrum, 30, 401430 (2008).

3. G. Buyukozkan, O. Feyzioglu, M.S. Ersoy, Evaluation of 4PL operating models: A decision making approach based on 2-additive Choquet integral. International Journal of Production Economics, 121, 112-120 (2009).

4. C. Kahraman, O. Engin, O. Kabak, I. Kaya, Information systems outsourcing decisions using a group decision-making approach. Engineering Applications of Artificial Intelligence, 22, 832-841 (2009).

5. S.P. Wan, F. Wang, L.L. Lin, J.Y. Dong, J.Y. An intuitionistic fuzzy linear programming method for logistics outsourcing provider selection. Knowledge-Based Systems, 82, 80-94 (2015).

6. K. Govindan, R. Khodaverdi, A. Vafadarnikjoo, A grey DEMATEL approach to develop third-party logistics provider selection criteria. Industrial Management \& Data Systems, 116, 690-722 (2016).

7. J. Wang, J.Q. Wang, H.Y. Zhang, A likelihoodbased TODIM approach based on multi-hesitant fuzzy linguistic information for evaluation in logistics outsourcing. Computers \& Industrial Engineering, 99, 287-299 (2016).

8. M.M. Rajaeian, A.C. Steel, M. Lane, A systematic literature review and critical assessment of modeldriven decision support for IT outsourcing. Decision Support Systems, 102, 42-56 (2017).

9. F. Herrera, L Martínez, A 2-tuple fuzzy linguistic representation model for computing with words. IEEE Transactions on Fuzzy Systems, 8(6), 746752 (2000).

10. F. Herrera, L. Martínez, A model based on linguistic 2-tuples for dealing with multigranular hierarchical linguistic contexts in multi-expert decision-making. IEEE Transactions on Systems, Man, and Cybernetics-Part B: Cybernetics, 31(2), 227-234 (2001).

11. O. Cordon, F. Herrera, I. Zwir, Linguistic modeling by hierarchical systems of linguistic rules. IEEE Transactions on Fuzzy Systems, 10(1), 2-20 (2002).

12. C.L Hwang, K.P. Yoon, Multiple attribute decision making: methods and applications, New York: Springer (1981). 